\title{
Rasgos de personalidad y asertividad en estudiantes de la Facultad de Ciencias de la Salud
}

\author{
Characteristics of personality and assertiveness in students of the Faculty of Health Sciences
}

\author{
Katterin Bermúdez Vargas ${ }^{1 *}$, Priscila Ccancce Gastelún ${ }^{2}$, Isaac Conde Rodríguez ${ }^{3}$
}

\begin{abstract}
RESUMEN
Objetivo: Determinar la relación entre los rasgos de personalidad y la asertividad en 210 estudiantes de ambos sexos, entre 16 y 25 años de edad del primer y segundo año de la Facultad de Ciencias de la Salud de una Universidad privada de Lima este. Materiales y Métodos: Este estudio fue de diseño no experimental, tipo cuantitativo, de alcance descriptivo correlacional, se utilizó el Inventario de Personalidad reducido en Cinco Factores (NEOFFI) y la Escala de Evaluación de la Asertividad (ADCA-1). Resultados: Los resultados mostraron que sí existe relación significativa entre las variable. De este modo, se encontró relación altamente significativa entre el rasgo neuroticismo con autoasertividad $(\mathrm{r}=-0.449 \mathrm{p}=0.000)$ y con heteroasertividad $(\mathrm{r}=-0.292 \mathrm{p}=0.000)$. Además, existe relación altamente significativa entre el rasgo extraversión con autoasertividad ( $\mathrm{r}=0.391 \mathrm{p}=0.000)$ y heteroasertividad $(r=0.198 \mathrm{p}=0.004)$. Conclusiones: Finalmente se halló relación altamente significativa entre el rasgo responsabilidad con autoasertividad $(r=0.447 \mathrm{p}=0.000)$ y con heteroasertividad $(\mathrm{r}=0.238 \mathrm{p}=0.001)$ en los estudiantes universitarios.
\end{abstract}

Palabras claves: Rasgos, personalidad, asertividad.

\begin{abstract}
Objective: To determine the relationship between the personality traits and the assertiveness in 210 students of both sexes, between 16 and 25 years of age of the first and second year of the Faculty of Health Sciences of a private University of East Lima. Materials and Methods: This was a non-experimental, quantitative, descriptive, correlational study. Five-Factor Personality Inventory (NEOFFI) and the Assertiveness Assessment Scale (ADCA-1) were used. Results: The results showed that there is a significant relationship between the variables. Thus, a highly significant relationship was found between the neuroticism trait with self-assertiveness $(r=-0.449$ $\mathrm{p}=0.000)$ and with heteroartivity $(\mathrm{r}=-0.292 \mathrm{p}=0.000)$. In addition, there is a highly significant relationship between the extraversion feature with self-assertiveness $(\mathrm{r}=0.391 \mathrm{p}=0.000)$ and heteroartivity $(\mathrm{r}=0.198 \mathrm{p}=$ 0.004). Conclusions: Finally, a highly significant relationship was found between the self-assertive responsibility $(\mathrm{r}=0.447 \mathrm{p}=0.000)$ and heteroartivity $(\mathrm{r}=0.238 \mathrm{p}=0.001)$ among university students.
\end{abstract}

Keywords: Traits, personality, assertiveness.

\footnotetext{
${ }^{1}$ Psicólogo, EP Psicología, Universidad Peruana Unión, Lima, Perú.

${ }^{2}$ Psicólogo, EP Psicología, Universidad Peruana Unión, Lima, Perú.

${ }^{3}$ Docente de Psicología, Facultad de Ciencias de la Salud, Universidad Peruana Unión, Lima, Perú.
} 


\section{INTRODUCCIÓN}

En el presente siglo, donde se apunta a la calidad total en los diferentes aspectos de la vida como el ambiente académico y laboral se considera como ciudadano de mundo aquel que sabe interactuar adecuadamente con sus semejantes; pues las personas por naturaleza son seres sociales y sensibles a la influencia del medio en el que se desarrollan (Grupo Banco Mundial, 2015). En este sentido, Caspi, Roberts y Shiner (2005) señalan que la forma en que los seres humanos se interrelacionan está determinada por sus rasgos de personalidad. Adicionalmente, Bartholomeu, Sancineto da Silva y Machado (2008) refieren que existen componentes de la personalidad, como la socialización que pueden favorecer o perjudicar el establecimiento de patrones de conducta para la interacción social.

Por su parte, Reyes (2003) señala que los rasgos de personalidad son producto de la interacción de la herencia con el ambiente, los cuales influyen en la estructura de la personalidad y en el curso de futuros aprendizajes, de este modo los individuos responden a situaciones complejas que pueden afectar sus experiencias con otras personas debido a que la persona necesita estar segura de sí misma y de sus capacidades específicas para expresar, sentimientos, opiniones y pensamientos en el momento oportuno, de la forma adecuada, considerando los derechos de los demás, las cuales son características que forman parte de la asertividad, la cual es definida por García y Magaz (2003) como una cualidad que se constituye en un acto de respeto e igualdad en relación a las cualidades y particularidades personales de cada ser humano al momento de establecer relaciones interpersonales.

En este sentido, Kirts (2011) encontró que los niveles altos de neuroticismo están asociados a la falta de asertividad, la cual genera complicaciones severas; tales como: timidez, baja autoestima e incluso podrían desarrollar ansiedad social en los estudiantes universitarios. Por otro lado, Peña (2005) manifiesta que la salud mental tiene relación con la asertividad, al señalar que el entrenamiento asertivo disminuye significativamente la ansiedad y el estrés. Respecto a esto, Castanyer (1996) señala que la asertividad es una habilidad muy conveniente en los profesionales de la salud, porque se requiere tratar con respeto al enfermo, no obstante, no se le puede conceder todo lo que quiera, el profesional de la salud tiene que saber decir no cuando haga falta y así vivirá más tranquilo.

Las diferentes teorías de la personalidad planteadas en el área proporcionan un marco de referencia dinámico de la personalidad que se manifiesta en la conducta humana. Es así que para la variable rasgos de personalidad, el presente estudio se basa en el modelo explicativo de las cinco dimensiones. Goldberg (1993) explicó en la década de los 70 la existencia de un modelo importante, llamado Los Cinco Grandes (Big Five) establecido a partir de la aproximación léxica e integración de los aportes de autores como Norman, Golberg, Peabody, Trapnell, Wiggins y Digman (1966).

Por lo tanto, Costa y McCrae (1992) definen que el modelo de la personalidad consiste en la manera que un individuo difiere en sus estilos individuales, emocionales, interpersonales y motivacionales, posteriormente estos estilos son resumidos en términos de cinco factores básicos llamados: neuroticismo $(\mathrm{N})$, extraversión $(\mathrm{E})$, apertura a la experiencia (O), agradabilidad (A) y responsabilidad (R). El modelo planteado por Costa y McCrae (1992) denominado bigfive, es un modelo comprensivo que fomenta la exploración de las interacciones entre la personalidad y otros fenómenos.

Además, para relacionar la variable rasgos de personalidad y asertividad, Eysenck (1987) señaló que las experiencias de vida y las formas de tomar las cosas e incluso la propia biología influyen sobre la timidez, todo esto es crucial en la raíz de los problemas de asertividad, pues algunas personas son más sensibles e inestables emocionalmente y por lo mismo más proclives a la ansiedad. Por lo tanto, los obstáculos que presentan las personas para crear una buena atmósfera de comunicación deriva de ellas mismas y se vincula con el concepto de sí mismo, además una óptima autoestima se relaciona con una comunicación funcional y asertiva; al mismo tiempo, una baja autoestima conlleva a una comunicación disfuncional y no asertiva (Naranjo, 2008).

Uno de los modelos teóricos que explica el aprendizaje de la asertividad es la teoría del aprendizaje social de Bandura, el cual toma en consideración la capacidad humana de pensar y planificar experiencias; pues las personas hacen planes y predicen las consecuencias de sus acciones, es así como manejan las diversas situaciones que se enfrenta en la vida diaria (Sollod, Wilson y Monte, 2009). Además, Bandura (2001), señaló que el individuo crea, modifica y destruye el entorno, y los cambios que introduce en el mismo afectan a su conducta y a la naturaleza de su vida futura.

Por otro lado, Cornejo (citado por Camacaro, 2011) añadió que un liderazgo asertivo, puede desarrollarse como cualquier otra habilidad humana y es susceptible 
de aprenderse, entrenarse y mejorarse. Utilizando técnicas como: identificar los estilos básicos de la conducta interpersonal, para reconocer la mejor forma de practicar el estilo asertivo; comparar las situaciones en las que se quiere ser más asertivos; describir las situaciones problemáticas; escribir un guión para el cambio de conducta, para afrontar la conducta de forma asertiva y desarrollar del lenguaje corporal adecuado, evitando manipular a los demás.

Por lo expuesto, el objetivo del estudio es determinar la relación que existe entre los rasgos de personalidad y la asertividad en estudiantes del primer y segundo año de la Facultad de Ciencias de la Salud de una Universidad Privada de Lima este.

\section{MATERIALES Y MÉTODOS}

Se contó con la participación de 210 estudiantes de la Facultad de Ciencias de la Salud, los cuales fueron seleccionados a través de un muestreo no probabilístico por conveniencia ya que se eligieron de acuerdo a las necesidades del estudio.

En cuanto a las características sociodemográficas de la muestra indican que el $71.9 \%$ de los estudiantes son mujeres. Respecto a la religión se encontró que el $84 \%$ son adventistas. Asimismo, el $42 \%$ de los evaluados se encuentran entre las edades de 21 a 25 años. Con respecto a las Escuelas Profesionales, el $57 \%$ son de la E.P. de psicología y el $60 \%$ son de la costa.

\section{Instrumentos}

El inventario de la personalidad NEO FFI fue creado por Costa y McCrae (1992) que ofrece una medida rápida de los cinco factores de la personalidad, los resultados se obtienen a través de niveles; alto, medio y bajo. Consta de 72 afirmaciones; las respuestas se dividen en 5 categorías que van desde totalmente en desacuerdo y termina en totalmente de acuerdo, adquiere una puntuación de cero y totalmente de acuerdo una calificación de cuatro en las preguntas directas, en el caso de las preguntas indirectas la forma de puntuar se invierte, es decir que totalmente en desacuerdo ahora obtiene una calificación de cuatro y totalmente de acuerdo de cero. El test está agrupado en 5 dimensiones, las cuales especificamos a continuación: Dimensión neuroticismo (1; 13; $19 ; 25 ; 31 ; 37 ; 43 ; 61 ; 7 ; 49 ; 55 ; 67)$; dimensión extraversión $(2 ; 8 ; 14 ; 26 ; 38 ; 56 ; 62 ; 20 ; 32 ; 44$; $50 ; 68)$; dimensión apertura $(3 ; 15 ; 21 ; 33 ; 39 ; 57$; $63 ; 9 ; 27 ; 45 ; 51 ; 69)$; dimensión amabilidad $(4 ; 28$; $34 ; 46 ; 52 ; 58 ; 10 ; 16 ; 22 ; 40 ; 64 ; 70)$; dimensión responsabilidad $(11 ; 17 ; 23 ; 29 ; 35 ; 41 ; 47 ; 53 ; 5$;
$59,65,71)$ cabe mencionar que el test cuenta con una escala de veracidad llamada distorsión a la cual pertenecen 12 ítems $(6 ; 12 ; 18 ; 24 ; 30 ; 36 ; 42 ; 48 ; 54$; $60 ; 66 ; 72)$.

Se calcularon los índices de confiabilidad, los cuales fueron estimados a través del método de consistencia interna por el coeficiente alfa de Cronbrach. Obteniendo un valor de 0.776 lo cual evidencia un alto nivel de confiabilidad. Para los efectos de la validez de contenido de la prueba, se realizó a través del criterio de jueces, para los cual se tomó en cuenta a cinco expertos en el área y en metodología de la investigación, quienes se desempeñan como docentes y especialistas en su área, encontrándose que el $100 \%$ de los ítems de la prueba son válidos. Para la validez de constructo se utilizó el análisis estadístico $\mathrm{R}$ de Pearson por el método de análisis ítems-test. Se encontró correlación significativa entre 3 dimensiones: neuroticismo (0.669), extraversión (0.77) y responsabilidad (0.68), mientras que en las dimensiones de apertura (0.55) y amabilidad (0.44) no se encontró correlación significativa. Se corroboró la calidad métrica del instrumento en la muestra estudiada, donde el inventario presenta una adecuada fiabilidad y validez a excepción de dos dimensiones: amabilidad y apertura, las cuales no fueron incluidos en el análisis estadístico correspondiente.

La Evaluación de la Asertividad ADCA-1, es un cuestionario autoaplicado que consta de 35 afirmaciones, de las cuales 20 preguntas corresponde a la dimensión asertividad y 15 a la dimensión heteroasertividad (García y Magaz, 2003).

El objetivo de la escala es proporcionar información sobre las dos dimensiones: asertividad y heteroasertividad. Esta prueba puede ser contestada por jóvenes y adultos de ambos sexos que tengan un nivel mínimo de instrucción de sexto grado de primaria, puede ser administrado de manera grupal o individual y debe realizarse en un ambiente apropiado que permita la concentración y comodidad. El tiempo de duración es de 20 a 30 minutos en promedio.

El ADCA-1 incluye un cuadernillo que contiene los ítems de la escala. En la primera página se consigna las instrucciones para contestar la prueba y las siguientes páginas contienen las 35 afirmaciones de las mismas, para las 35 afirmaciones se utilizó la escala Likert que va desde Totalmente en desacuerdo (TD), desacuerdo (D), Ni de Acuerdo Ni Desacuerdo (ND), de acuerdo (A), y totalmente de acuerdo (TA). Para la calificación se debe tomar en cuenta que el estudiante haya respondido de manera honesta, precisa sin dejar preguntas sin contestar. 
Respecto a la validez de la Escala de evaluación de la asertividad (ADCA-1), fue administrado en 254 personas, 127 de población clínica y los otros de población general. Este instrumento ha sido creado en el año 2003 por (García y Magaz, 2003), en la ciudad de Lima. Asimismo la confiabilidad fue obtenido por el coeficiente de consistencia interna autoasertividad $\mathrm{N}=1.345, \mathrm{R}=0.91$ y heteroasertividad $\mathrm{N}=1.323, \mathrm{R}=$ 0.82 y ha sido sometido a la validez criterial, validez de discriminante y validez concurrente.
Para el análisis estadístico se utilizó el software estadístico SPSS versión 22.0 para Windows. Una vez recolectada la información se transfirió a la matriz de datos del software estadístico para su respectivo análisis, los resultados obtenidos han sido ordenados en tablas. Se llevó a cabo el proceso de la distribución normal y se determinó usar el estadístico $r$ de Pearson para analizar la relación.

\section{RESULTADOS}

Tabla 1

Coeficiente de correlación entre rasgos de personalidad y asertividad.

\begin{tabular}{lcccc}
\hline & \multicolumn{2}{c}{ Autoasertividad } & \multicolumn{2}{c}{ Heteroasertivida } \\
\cline { 2 - 5 } Rasgos de personalidad & $r$ & $p$ & $r$ & $p$ \\
\hline Neuroticismo & $-0.449^{* *}$ & 0.000 & $-0.292^{* *}$ & 0.000 \\
Extraversión & $0.391^{* *}$ & 0.000 & $0.198^{* *}$ & 0.004 \\
Responsabilidad & $0.447^{*}$ & 0.000 & $0.238^{* *}$ & 0.001 \\
\hline
\end{tabular}

** La correlación es significativa al nivel 0.01

En la Tabla 1, se aprecia el coeficiente de correlación de Pearson; indica que existe una relación negativa moderada y altamente significativa entre neuroticismo y autoasertividad $(r=-0.449 \mathrm{p}=0.000)$, con respecto a neuroticismo y hetoroasertividad se halló que mantienen una correlación inversa baja pero altamente significativa $(\mathrm{r}=-0.292 \mathrm{p}=0.000)$; es decir, a menor nivel de neuroticismo, mayor nivel de autoasertividad y heteroasertividad. Además, existe correlación moderada y altamente significativa entre extraversión y autoasertividad $(r=0.391 \mathrm{p}=0.000)$, mientras que se halló correlación baja pero altamente significativa entre extraversión y hetoroasertividad $(\mathrm{r}=0.198 \mathrm{p}=0.004)$.

Finalmente, el coeficiente $r$ de Pearson muestra una correlación moderada y altamente significativa entre la dimensión autoasertividad y responsabilidad $(\mathrm{r}=0.447$ $\mathrm{p}=0.000$ ), sin embargo, la dimensión responsabilidad tiene una relación baja pero altamentente significativa respecto a la dimensión heteroasertividad $(\mathrm{r}=0.238$ $\mathrm{p}=0.001)$.

Tabla 2

Prueba de bondad de ajuste a la curva normal para las variables de estudio.

\begin{tabular}{llllll}
\hline Instrumentos & Variables & Media & D.E. & K-S & $p$ \\
\hline & Neuroticismo & 18.11 & 6.49 & 0.77 & 0.09 \\
& Extraversión & 30.91 & 7.45 & 0.72 & 0.66 \\
Rasgos de la & & & & & \\
personalidad & Responsabilidad & 30.28 & 6.83 & 1.0 & 0.58 \\
& Autoasertividad & 62.32 & 9.61 & 1.2 & 0.67 \\
Asertividad & Heteroasertividad & 44.79 & 8.03 & 0.72 & 0.24 \\
${ }^{*} \mathrm{p}<0.05$ & & & & & \\
\hline
\end{tabular}

Con el propósito de realizar los análisis comparativos y contrastar las hipótesis planteadas, se ha procedido a realizar primero la prueba de bondad de ajuste para precisar si las variables presentan una distribución normal. En ese sentido, la Tabla 2 presenta los resultados de la prueba de bondad de ajuste de Kolmogorov-Smirnov (K-S); como se puede observar, también, los datos correspondientes a las 5 dimensiones en su mayoría presentan una distribución normal dado que el coeficiente obtenido (K-S) es 
significativo $(\mathrm{p}<0.05)$. Por tanto, para los análisis estadísticos correspondientes se empleará estadística no paramétrica.

\section{DISCUSIÓN}

El objetivo de este estudio fue analizar la relación entre los rasgos de personalidad y asertividad en estudiantes del primer y segundo año de la Facultad de Ciencias de la Salud de una Universidad Privada de Lima este, los resultados mostraron que sí existe relación significativa entre estas variables, puesto que las dimensiones de rasgos de personalidad se vinculan con las dimensiones de asertividad. Estos hallazgos coinciden con el trabajo realizado por Kirts (2011) en estudiantes, quien encontró relación altamente significativa entre las variables y explica que la asertividad es una habilidad de comunicación aprendida que se relaciona con los diversos factores de la personalidad.

En cuanto a la relación entre el rasgo neuroticismo y la autoasertividad en estudiantes, el coeficiente $r$ de Pearson muestra una relación negativa moderada y altamente significativa entre las variables $(r=-0.449$, $\mathrm{p}=0.000$ ), es decir, que a menor nivel de neuroticismo en los participantes se incrementarán las habilidades para enfrentar sentimientos de inferioridad y pensamientos aterradores los cuales impiden al individuo que exprese opiniones, además niveles elevados en autoasertividad, denotan fortaleza en la capacidad del control de emociones que contribuye a disminuir el estrés y la ansiedad. Este resultado concuerda con el trabajo realizado por Kirts (2011) quien determinó que sí existe relación entre el rasgo neuroticismo y autoasertividad en estudiantes, a bajos niveles de neuroticismo, los estudiantes desarrollan la habilidad para afrontar conflictos interpersonales; es decir, enfrentan, miedos, temores y timidez, lo que les permite expresar sus ideas y defender sus derechos. Asimismo, Costa y McCrae (1992) refirieron que las personas con bajos niveles de neuroticismo suelen tener una respuesta emocional controlada, proporcionada, tranquila y despreocupada. Por su parte, Torres y Rodríguez (2010) mencionaron los niveles bajos de neuroticismo ayudan a enfrentar situaciones estresantes y combatir sentimientos negativos como los sentimientos de inferioridad y pensamientos aterradores que están vinculados a la manera adecuada de expresar las ideas.

Respecto a la relación entre el rasgo neuroticismo y la heteroasertividad en estudiantes, se encontró que existe relación débil pero altamente significativa entre las variables mencionadas $(r=-0.292 \mathrm{p}=0.00)$ lo que significa que las personas con altos niveles de neuroticismo se caracterizan por mostrar sensibilidad a la crítica, falta de confianza en sí mismo, pobre ajuste emocional y afrontamiento al estrés, así como dificultades para interactuar adecuadamente con los demás, además quienes puntúan bajo en heteroasertividad, implica que evidencian dificultades para reconocer que los demás tienen derecho a hacer respetar sus derechos. En este sentido, Eysenck (1985) y Machicao (2012) arguyen que los estudiantes con mayores puntajes en neuroticismo presentan ansiedad frente a las situaciones ambientales y se perciben incapaces de afrontar los problemas, lo que genera desorganización emocional, desesperanza y actitudes negativas. Por lo tanto, les cuesta reconocer que los demás tienen derecho a decir no ante una petición, a tener una posición diferente y a mostrar malestar cuando no están de acuerdo (García y Magaz, 1994). Además, León, Rodríguez, Ferrel y Ceballos (2009) señalaron que el desarrollar la heteroasertividad en los estudiantes de la Facultad de Salud ayudará a controlar mejor la ansiedad, ya que la persona se vuelve más espontánea y segura.

En cuanto a la relación entre el rasgo extraversión y la autoasertividad en estudiantes, el coeficiente $r$ de Pearson muestra una relación moderada y altamente significativa entre las variables mencionadas $(\mathrm{r}=$ $0.391 \mathrm{p}=0.00$ ), es decir que el rasgo extraversión está relacionada con la autoasertividad, puesto que las personas que tienen la facilidad para entablar relaciones interpersonales, son animosas y alegres, además tienen la capacidad de expresar sus opiniones respetando a los demás.

Este resultado concuerda con el trabajo realizado por Bratko, Vukosav, Zarevski y Vranic (2002) quienes hallaron que niveles bajos de extraversión están relacionados con la timidez, mientras que niveles altos de extraversión están vinculados con la dimensión de la autoasertividad; es decir que los estudiantes que suelen ser expresivos y sociables, presentan también una adecuada capacidad para hacer respetar sus opiniones. Asimismo, se puede hallar una explicación teórica según Eysenck (1987) quien explica que niveles altos en extraversión suelen estar coligado a personas sociables, despreocupadas y entusiastas. Del mismo modo, Costa y McCrae (1992), añadieron que las personas con alta extraversión suelen ser asertivos, activos y poseen muchos amigos. Lo expuesto coincide con el trabajo realizado por Cassaretto (2009) donde se halló que las personas con puntuaciones altas en extraversión tienen mayor confianza en sí mismas, les gusta estar con amigos disfrutan estar más tiempo con ellos que estar solos y manifiestan opiniones sin miedo a la crítica y al rechazo. 
Respecto a la relación entre el rasgo extraversión y la heteroasertividad en estudiantes del primer y segundo año de la Facultad de Ciencias de la Salud de una universidad privada de Lima este, se encontró que existe relación débil pero altamente significativa entre las variables mencionadas $(r=0.198 \mathrm{p}=0.000)$, lo que a mayores niveles de extraversión mayores serán los niveles de heteroasertividad, esto significa que cuando una persona es espontánea y expresiva podrá llevar a cabo una mejor interacción social que contribuirá a desarrollar la capacidad de comprensión hacia los derechos ajenos, característica principal de la heteroasertivo. Hogan, Johnson y Briggs (1997), afirmaron que la extraversión es un factor que evalúa la cantidad e intensidad en las relaciones sociales, pues permite observar el nivel de actividad, la capacidad para experimentar placer y otras emociones positivas, asimismo, los sujetos con alta extraversión son vistos como asertivos, activos, enérgicos, locuaces, animosos, optimistas lo que facilita las interacciones sociales, pues presentan mayor capacidad de persuasión, y alta dedicación en la obtención de metas. Asimismo, Costa y McCrae (1992), coincidieron en señalar que la extraversión facilita el dominio y seguridad que una persona muestra al expresarse en espacios grupales. Además, Ruiz (2014) señaló que un individuo con características heteroasertivas, sabe cuándo y cómo dirigirse a los demás, a mantener el debido respeto ante las limitaciones personales, entendiendo y asimilando la diversidad de ideas, propuestas y errores. De este modo, se hace evidente que a niveles altos de extraversión habrá más facilidad para desarrollar la heteroasertividad.

En cuanto a la relación entre el rasgo responsabilidad y la autoasertividad en estudiantes, el coeficiente $r$ de Pearson muestra una relación moderada y altamente significativa entre las variables mencionadas $(r=$ $0.447 \mathrm{p}=0.00$ ), lo que significa que los participantes logran alcanzar objetivos y metas propuestas de manera ordenada, también está relacionada al hecho de asumir las consecuencias que implican no terminar una tarea. Este resultado concuerda con el trabajo realizado por Contreras, Espinoza y Esguerra (2008) quienes hallaron correlación entre el rasgo responsabilidad y la autoasertividad en estudiantes, encontraron que el ser responsable implica ser perseverante, estar motivado y tener confianza en sí mismo, además refieren que las personas deben atreverse hacer lo que sienten y desean para así lograr el equilibrio permanente que es reflejado en la conducta positiva de la persona. Asimismo, Costa y McCrae (1992) encontraron que las personas con niveles altos en el rasgo responsabilidad suelen ser honestos e inteligentes, pues se puede confiar en ellos, ya que siempre van a cumplir con sus actividades programadas. Además, Macías y Camargo (2013) y Flores (2004) consideran que las personas con niveles altos en autoasertividad buscan desarrollar destrezas asertivas, pues tienen la habilidad para planificar y llevar a cabo una tarea programada, de esta manera manifiestan que el ser responsable implica tener una conducta asertiva, ya que la persona tendrá la capacidad de mostrar seguridad en sí mismo para decir no cuando se sabe que no se va cumplir con actividades programadas, porque ya tienen trabajos pendientes, lo que muestra un elevado respeto hacia sí mismo.

Respecto a la relación entre el rasgo responsabilidad y la heteroasertividad en estudiantes, se encontró que existe relación débil pero altamente significativa entre las variables mencionadas $(r=0.238 p=0.001)$ es decir, que mientras mayores sean los niveles de responsabilidad, mejores serán los niveles de heteroasertividad. En este sentido, Costa y McCrae (1999), señalaron que las personas que presentan características del rasgo responsabilidad son trabajadoras, confiables, cumplidas y decididas, asimismo muestran comprensión hacia los que no tienen las mismas habilidades que ellos y piensan cuidadosamente antes de actuar, lo cual es un indicador de respeto; por lo que se relaciona con la heteroasertividad, pues García y Magaz (1994) señalan que esta dimensión consiste en respetar los derechos de los demás.

\section{Declaración de financiamiento y de conflicto de intereses:}

El estudio fue financiado por los autores, quienes declaran no tener algún tipo de conflicto de interés en la investigación realizada.

\section{Correspondencia}

Katterin Bermudez Vargas

e-mail: katterin.bermudez.vargas@gmail.com 


\section{REFERENCIAS BIBLIOGRÁFICAS}

Bandura, A. (2001). Personal communication. New York: Holt, Rinehart and Winston Recuperado de: http:// cogweb.ucla.edu/crp/Media/Bandura_01.pdf.

Bartolomé, Sancineto da Silva y Machado (2088). Traços de personalidade e habilidades sociais em universitarios, Psico-USF, 13(1), pp. 41-50. Recuperado de: http:// pepsic.bvsalud.org/pdf/psicousf/v13n1/v13n1a06.pdf

Bratko, V., Vukosav, E., Zarevski, K. y Vranic, A. (2002). Five factor model and shyness and assertivenes. Review of Psychology, 9(2), 17-23. Recuperado de: http:// mjesec.ffzg.hr/revija.psi/vol\%2009\%20no\%201-2\%20 2002/Bratko_2002_9-1-2.pdf

Camacaro, Y. (17 de noviembre del 2011). Orientación cognitivo conductual para el ejercicio de un liderazgo asertivo de los gerentes educativos en la nueva era. Revista científica digital del centro de investigación y estudios gerenciales, 2(2), pp. 85-95. Recuperado de: http://www.grupocieg.org/archivos revista/2-2-7\%20 ( $85-95) \% 20 \mathrm{Camacaro} \% 20 \mathrm{Yris} \% 20 \mathrm{rcieg} \% 20$ noviembre\%2011_articulo_id72.pdf

Cassaretto, M. (2009). Relación entre las cinco grandes dimensiones de la personalidad y el afrontamiento en estudiantes preuniversitarios de Lima Metropolitana. (Tesis de Posgrado en Psicología). Universidad Nacional Mayor de San Marcos Lima, Lima-Perú. Recuperado de: http://cybertesis.unmsm.edu.pe/bitstream/ cybertesis/619/1/Cassaretto_bm.pdf.

Caspi, A., Roberts, B. y Shiner, L. (2005). Personality devolopment: Stability and Change. Annual Review of Psychology, 56(1), 453-484.

Costa, P. y McCrae, R. (mayo, 1997). Personality trait structure as a human universal. APA PsycNET Direct,52(5), 509516. Recuperado de: http://psycnet.apa.org/index. cfm?fa=buy.option ToBuy\&id=1997-04451-001.

Castanyer, O. (1996). La asertividad: expresión de una sana autoestima. Bilbao, Desclée de Brouwer.

Contreras, F., Espinoza, J., Esguerra, G. (2008). Personalidad y afrontamiento en estudiantes universitarios. Revista de Psicología de Bogotá, 8(2): 1657-9267. Recuperado dehttp://www.scielo.org.co/pdf/rups/v8n2/v8n2a02.pdf.

Eysenck, H. (1987). Personalidad. Londres. Inglaterra: Mac Millan.

García, M. y Magáz, A. (1993). ADCA-1 Escala de evaluación de la asertividad. Manual técnico. Madrid, España: CEPE.

Goldberg, L. (1993). The Structure of Phenotypic Personality Trait. Reviews American Psychologist, 48(1), 2634. Recuperado de: http://psycnet.apa.org/journals/ amp/48/1/26/.

Grupo Banco Mundial. (2015). Informe sobre el desarrollo mundial. Programa general mente sociedad y conducta. Recuperado de: www.worldbank.org.

Hogan, R. Johnson, J. Briggs, S. (1997). Handbook of Personality Psychology. Academic Press: San Diego CA

Kirts, L. (2011). Investigating the relationship between assertiveness and Personality characteristic. Thesis in Psychology. Orlando, Florida: University of Central Florida. Recuperado de: http://etd.fcla.edu/CF/ CFH0004071/Kirst_Laura_K_201205_BS.pdf

León, A., Rodríguez, C., Ferrel, R. y Ceballos, A. (2009). Asertividad y autoestima en estudiantes de primer semestre de la Facultad de Ciencias de la Salud de una universidad pública de la ciudad de Santa Marta. Colombia. Psicología desde el

Caribe, 24 (25), pp15-28. Recuperado de: http://rcientificas. uninorte.edu.co/index.php/psicologia/article/ view/615/5354.

Machicao, R. (2012). Factores de personalidad y actitud hacia los residuos sólidos en estudiantes de una universidad particular de Lima, Perú. Revista Peruana de Psicología, 7(1), 1-2. Recuperado de: http://www.upch.edu.pe/ vrinve/dugic/revistas/index.php/RPH/article/view/2260.

Macías, E. y Camargo, G. (2013). Corporación universitaria minuto de Dios facultad de ciencias de la comunicación especialización en comunicación educativa. Bogotá: Corporación Universitaria Minuto de Dios. Recuperado de: http://repository.uniminuto. edu:8080/jspui/bitstream/10656/2852/1/TECE_Camar goReinosoGina_2014.pdf

Naranjo, M. (2008). Relaciones interpersonales adecuadas mediante una comunicación y conducta asertivas. Revista electrónica de Educación, 8(1): 1-27. Recuperado de http://revista.inie.ucr.ac.cr

Norman, W., Golberg, P., Peabody, J., Trapnell, P., Wiggins, E. y Digman, E. (1966). Rates and randomness in personality. Review of Psychology, 4(1), 681-691.

Peña, M. (2005). Niveles de asertividad en los enfermos y personal técnico, y la satisfacción de los pacientes en los servicios de salud del hospital Sergio Bernales. (Tesis de maestría en administración en servicios de salud). Universidad Nacional Federico Villarreal. Lima.

Reyes, Y. (2003). Relación entre rendimiento académico, ansiedad ante los exámenes, rasgos de personalidad, autoconcepto y asertividad en estudiantes del primer año de psicología de la UNMSM. (Tesis de licenciatura en Psicología). Universidad Nacional Mayor de San Marcos, Lima. Recuperado de: http://sisbib.unmsm.edu. pe/bibvirtualdata/tesis/salud/reyes_t_y/t_completo.pdf.

Ruiz, N. (2014). Satisfacción laboral y las dimensiones de asertividad en operarios de una organización privada de hidrocarburos Talara-Piura 2012 (Tesis de licenciado en psicología). Recuperado de: http://tesis.usat.edu.pe/ jspui/bitstream/123456789/329/1/TL_Ruiz_Bravo_ Portoc arrero_Norka.pdf

Sollod, R., Wilson, J. y Monte, C. (2009). Teorías de la Personalidad: Debajo de la máscara. México: McGrawHill/Interamericana de Editores.

Torres N., Rodríguez, J. (2010) Personalidad, aprendizaje y rendimiento. Revista electrónica elsevier, 2(8): 193201. Recuperado de http://riem.facmed.unam.mx/sites/ all/archivos/V2Num04/04_AO_PERSONALID AD_ APRENDIZAJE.PDF

Recibido: 19/02/2017

Aceptado: 02/05/2017 